\title{
Modification of aluminum alloy surface properties by wave-long laser texturing
}

\author{
Kseniya Batishcheva $^{1, *}$, Dmitriy Feoktistov ${ }^{1}$, Yuliya Shanenkova $^{1}$, and Ekaterina \\ Kirichenko ${ }^{2}$ \\ ${ }^{1}$ National Research Tomsk Polytechnic University, 634050 Tomsk, Russia \\ ${ }^{2}$ Institute of Thermophysics Siberian Branch Russian Academy of Sciences, 630090 Novosibirsk, \\ Russia
}

\begin{abstract}
Change of the static contact angle (SCA) of $10 \mu \mathrm{L}$ distilled water droplet on laser textured aluminium alloy (AMG-6) substrates was studied. The texture was deposited by a laser system based on a fiber laser. An increase in the power of laser radiation is found to lead to a decrease in the SCA measured on the first day after texturing. Change of dispersion and polar surface energy components of textured substrates is determined. Under the influence of the environment, the surface properties of AMG-6 change with time. SCA increased and reached stable state on the twentieth day after texturing.
\end{abstract}

\section{Introduction}

Control of wetting attracts the researchers' attention [1-4] because of the possibility of a wide range of applications: the creation of self-cleaning, antimicrobial, anti-corrosion, waterproof materials, reducing frictional forces, surface resistance. Research in this area is of interest to enterprises engaged in the development of equipment for transportation, separation of liquids, sounding devices, antennas, and radars in the space industry, in the design of satellites.

Currently the ways to create surfaces with controlled properties are under search. When choosing a method, decisive factors are the cost-effectiveness of the installation, ease of use, the possibility of obtaining samples with different texture, roughness. The following methods of texturing are known:

1 Chemical deposition from gas phase and sublimation [5]

It is a method of obtaining nanotubes or nanorods arrays by means of their deposition from the gas phase (chemical vapor deposition, CVD). This method can produce ordered carbon nanotubes arrays of the same diameter, a texture matrix that simulating the surface of the lotus leaf. To make such surface a superhydrophobic, an additional chemical modification of the surface is necessary. In addition to carbon nanotubes, the method makes it possible to obtain surfaces on the basis of soft silicone films without the need for further hydrophobization. A related method based on the deposition of matter from the gas phase is

\footnotetext{
*Corresponding author: bka1801@mail.ru
} 
sublimation, but unlike CVD, completely unordered rough surfaces forming a porous texture are formed after sublimation.

2 Electrochemical methods [6]

The method is used to deposit clusters of metals or metal oxides. The roughness of the resulting surface depends on the deposition time and the applied voltage. In [6], a superhydrophobic surface was obtained by electrochemical deposition of gold clusters on a multilayer polyelectrolyte matrix.

3 Methods based on the use of sol-gel materials [7]

To obtain porous textures with a high roughness, sol-gel materials based on metal alkoxides are used [7]. Controlling hydrolysis and condensation during the formation of the sol makes it possible to control the process of obtaining foams, and, consequently, the roughness of the obtained surfaces.

4 Template methods [8]

Membranes with an ordered system of one-dimensional pores of various diameters are used. Such an array is anodic aluminum oxide [8]. As precursors, which crystallize in the form of nanofibers inside such pores, polymers are used, for example, polyvinyl acetate or polyacrylonitrile. The main disadvantage of this method is the limitation of the maximum pore diameter.

5 Chemical modification of the surface [9]

Usually textured surfaces without further processing are not hydrophobic since their material is hydrophilic. Based on Wenzel and Cassie models, additional texturing of the surface will strengthen their hydrophilic properties. Depending on the material from which the surface consists, there are several options for hydrophobization. The deposition of an additional hydrophobic layer can occur by chemical modification of the surface with the necessary molecules through the gas phase in solution or by physical adsorption. Depending on the material, hydrophobic derivatives of alkanedioles, silanes, fatty acids, and others are used to hydrophobize the surface.

6 Reactive ion etching [10]

It is based on the use of reactive plasma etching material unprotected chemically. Part of the surface which does not need to be etched is chemically protected with coatings. Plasma is formed in a vacuum under the influence of an electromagnetic field. The type and amount of used gas depends on the material to be etched. For example, plasma based on sulfur hexafluoride is usually used for silicon surfaces.

7 Double texturing [11]

It is based on the production of superhydrophobic surfaces combining two textures - in micro- and nanoscale. The surfaces allow to increase the contact angle of wetting, to reduce the contact angle hysteresis, and also to increase the stability of the Cassie state. Microtexture determines the functional properties of the surface, for example, the length of sliding. Nanotexture is used to improve the wetting of surfaces.

8 Lithographic methods [12]

They are based on the use of templates. In other words, the topology of the surface is predetermined by the template form embedded in the control program: when etched by an electron beam; mask printed in advance for photolithography; a solid template which form is copied to another material by printing.

9 Laser irradiation [13]

It is based on a change in the surface morphology by the action of pulsed laser radiation. The deposition of laser radiation on the surface makes it possible to control wetting and spreading. By changing the parameters of the laser (power, diameter of the laser beam, and ablation rate of the substance from the surface), it is possible to create several samples with controlled hydrophobic and hydrophilic properties from one material. 
Based on the results of analysis of known methods for creating surfaces with controlled properties, the most promising is laser radiation. It has several advantages in comparison with others: non-contact, low-cost, simple and cheap in operation, with the possibility to obtain a wide range of textures (limited only by the parameters of the laser installation) and to process various materials.

The purpose of this work is to experimentally determine the dependence of the static contact angle on time on the treated aluminum surfaces by the laser radiation.

\section{Materials and methods}

Scheme of an experimental setup is presented in Fig. 1.

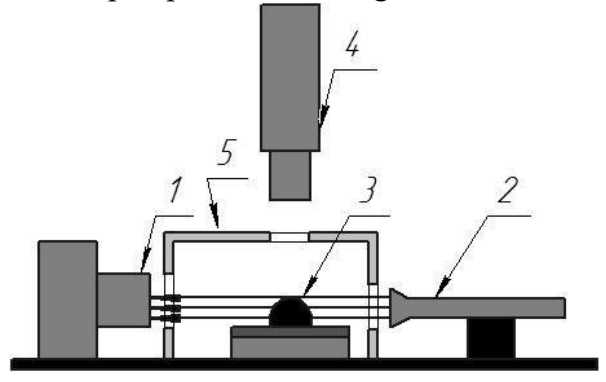

Fig. 1. Scheme of an experimental setup: 1 - photographic camera; 2 - source of plane parallel light; 3 - test cell, 4 - video camera; 5 - transparent box.

The main elements of setup: photographic camera Nikon D7100 with lens "Sigma AF $105 \mathrm{~mm}$ f/2.8 EX DG OS HSM MacroNikon F" 1; source of plane parallel light 2; test cell 3 consisting of goniometer to level the surface relative to the horizontal in two planes and the substrate; high-speed video camera Fastvideo-500M with lens "Nikon $85 \mathrm{~mm} \mathrm{f/1.4D} \mathrm{AF}$ Nikkor" with a shooting speed up to 500 frames per second and a resolution of 1280 x 1024 pixels 4; transparent box 5. The setup includes an optical shadow system. A detailed description of the operational principles is given in [14-15].

The experiment was conducted using six substrates made of aluminum alloy AMG-6. Their surfaces were polished. After polishing five substrates were treated with laser radiation, one remained unchanged. The texture is deposited with "Mini Marker 2 M 20" laser system. Table 1 shows the main technical characteristics of the laser system used for aluminum surfaces treatment.

Table 1. Technical characteristics of "Mini Marker 2 M 20" laser system used for surface texturing.

\begin{tabular}{|c|c|}
\hline Frequency of the laser beam, $\mathrm{Hz}$ & 99 \\
\hline Travel speed of the laser beam, $10^{-3} \mathrm{~m} / \mathrm{sec}$ & 800 \\
\hline Power of the laser radiation, $\mathrm{W}$ & $2,6,10,14,18$ \\
\hline
\end{tabular}

The average arithmetic deviation of the surface profile of the substrates ( $\mathrm{Ra}$ ) was determined on "HOMMEL TESTER T1000" profilometer. Photos of the microstructure were obtained on "Hitachi-3000M" microscope. All samples were examined by X-ray diffractometry using a Shimadzu XRD 7000S diffractometer. The polar and dispersion components of the surface energy of the substrates were determined.

A drop of distilled water with a volume of $10 \mu \mathrm{l}$ was placed on the surface by a highprecision dispenser. The SCA was measured for twenty days with an interval of five days.

\section{Results and discussion}


The SCAs values of laser textured substrates were obtained (Fig. 2).

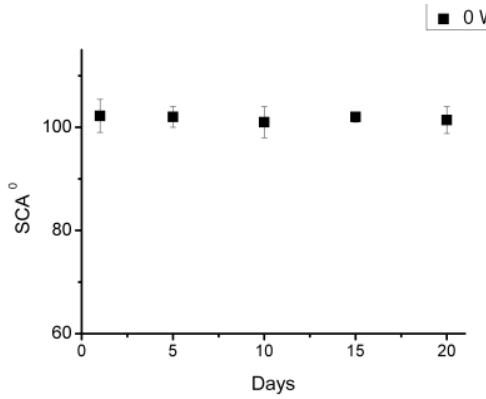

(a)

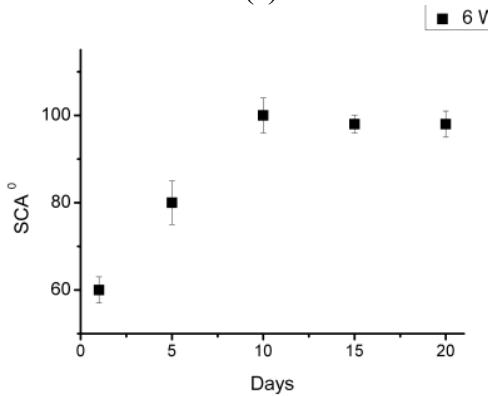

(c)

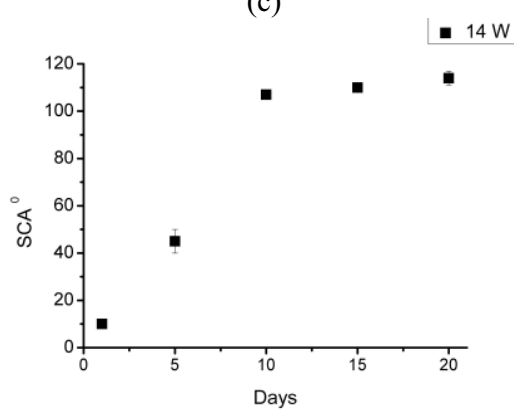

(e)

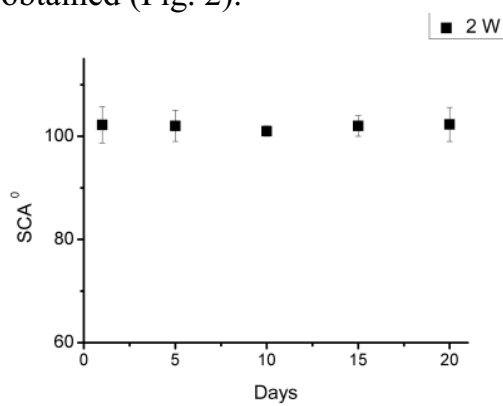

(b)

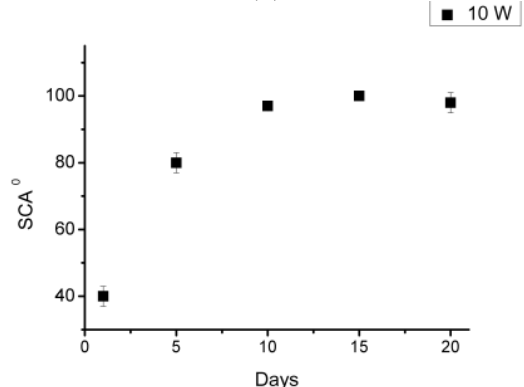

(d)

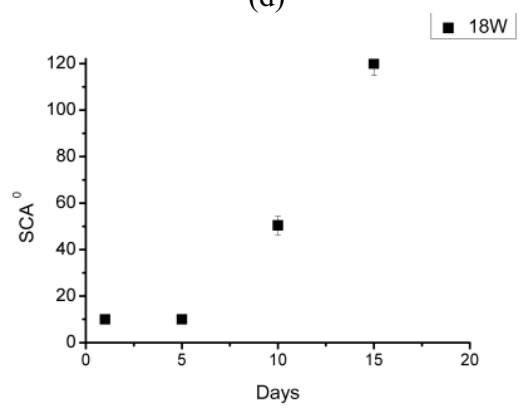

(f)

Fig. 2. Dependencies of SCAs on time after (a) - polishing surface; (b)-(f) - texturing by laser with a power of $2,6,10,1418 \mathrm{~W}$, respectively.

The SCAs values of laser textured substrates are obtained. Texturing by laser with a power of up to $2 \mathrm{~W}$ does not lead to a change in the SCA. Its value is equal to the value of the polished surface $\left(102^{\circ}\right)$. The surface energy remains constant $34 \pm 4 \mathrm{mN} / \mathrm{m}$. The effect of laser radiation with a power of up to $2 \mathrm{~W}$ does not affect the crystal lattice structure of the surface layer of the metal. The SCA is stable over time.

The SCA of laser textured substrates with a power of $6,10,14,18 \mathrm{~W}$ increased and reached a stable state after twenty days. An increase in the power of laser radiation is found to lead to a change in the structure of the crystal lattice. Twenty days later the SCA of laser treated substrates with a power of $6,10 \mathrm{~W}$ reached the value of a polished surface $\left(102^{\circ}\right)$. The laser treatment with a power of $14,18 \mathrm{~W}$ made it possible to obtain a porous texture formed by a network of crystals of various sizes and shapes. The depth of the pores does not exceed $2 \pm 0.19 \mu \mathrm{m}$. SCAs were $114^{\circ}$ and $120^{\circ}$, respectively. Over time the sessile drop under the action of gravity spread out filling the pores. SCA decreased to $70^{\circ}$. 


\section{Conclusions}

It has been found that the value of the SCAs of laser textured substrates with power of 6 , $10,14,18 \mathrm{~W}$ varies with time. A stable condition occurs on the twentieth day after laser texturing. The increase in the SCA is due to a change in the crystal lattice and a change in the ratio of the polar and dispersion components of the surface energy of the substrates. Laser texturing with power of $14,18 \mathrm{~W}$ is found to improve the hydrophobic properties of aluminum alloy AMG-6 surface.

The reported study was supported by the grant of President of Russian Federation for the government support of young Russian scientists (MK-6810.2016.8).

\section{References}

1. D. Zaitsev, D. Kirichenko, O. Kabov, Technical Physics Letters, 41, 6 (2015)

2. S. Misyura, Appl. Therm. Eng. 113, 472-480 (2017)

3. A. Sivkov, A. Ivashutenko, Y. Shanenkova, I. Shanenkov, Adv. Powder Technol. 27, 4 (2016)

4. R. S. Volkov, G. V. Kuznetsov, J. C. Legros, P. A. Strizhak, Int J Heat Mass Transf. 95, 184 (2016)

5. K. Lau, J. Bico, K. Teo, M. Chhowalla, G. Amaratunga, W. Milne, G. McKinley, K. Gleason, NanoLetters, 3, 12 (2003)

6. X. Zhang, F. Shi, X. Yu, H. Liu, Y. Fu, Z. Wang, L. Jiang, X. Li, J. Am. Chem. Soc. 126, 10 (2004)

7. N. Shirtcliffe, G. McHale, M. Newton, C. Perry, Langmuir, 19, 14 (2003)

8. L. Feng, S. Li, H. Li, J. Zhai, Y. Song, L. Jiang, D. Zhu, Angew. Chem. Int. Ed. 41, 7 (2002)

9. Z. Guo, F. Zhou, J. Hao, W. Liu, J. Am. Chem. Soc. 127, 45 (2005)

10. C. Pakpum, N. Pussadee, Surf. Coat. Technol. 306, 194-199 (2016)

11. N. Patankar, Langmuir, 20, 19 (2004)

12. H. Zhao, K. Park, K. Law, Langmuir, 28, 42 (2012)

13. F. Shi, X. Chen, L. Wang, J. Niu, J. Yu, Z. Wang, X. Zhang, Chem. Mater. 17, 24 (2005)

14. G. Kuznetsov, D. Feoktistov, E. Orlova, J Eng Phys Thermophy, 89, 2 (2016)

15. G. Kuznetsov, D. Feoktistov, E. Orlova, K. Batishcheva, Colloid J. 78, 3 (2016) 